\title{
A importância do exame Preventivo de Câncer de Colo de Útero e os fatores relacionados a não adesão
}

The importance of cervical cancer screening and factors related to non-adherence

La importancia del cribado del cáncer de cuello uterino y los factores relacionados con la falta de adherencia

Thaís Rodrigues de Sousa Silva ORCID: https://orcid.org/0000-0002-7798-4461 Universidade CEUMA, Brasil E-mail: thais_itz@hotmail.com

Jéssica Costa Moreira dos Santos ORCID: https://orcid.org/0000-0001-7936-8497 Universidade CEUMA, Brasil

E-mail: jessiica_costta@hotmail.com Jayne Sousa de Oliveira ORCID: https://orcid.org/0000-0003-3366-8544 Universidade CEUMA, Brasil

E-mail: Jaynesousadeoliveira@hotmail.com

Vitor Pachelle Lima Abreu

ORCID: https://orcid.org/0000-0001-9065-3272

Universidade Federal do Tocantins, Brasil E-mail: vpachelle@gmail.com

Rosivane Rodrigues da Silva ORCID: https://orcid.org/0000-0001-7119-8526 Universidade Federal do Maranhão, Brasil

E-mail: enfermeirarose9@gmail.com

Kassyo Lenno Sousa Dantas ORCID: https://orcid.org/0000-0001-8191-6923 Universidade CEUMA, Brasil

E-mail: kassyolenno@outlook.com

Robson Mariano Oliveira Silva

ORCID: https://orcid.org/0000-0002-3654-5278

Universidade Federal do Maranhão, Brasil

E-mail: robsonmariano01@hotmail.com

Palloma Oliveira da Silva Januário ORCID: https://orcid.org/0000-0002-8220-4175

Universidade Federal do Maranhão, Brasil

E-mail: palloma.oliversilva@hotmail.com

Gysllayne Fernandes de Sousa Gonçalves

ORCID: https://orcid.org/0000-0001-9212-4550

Universidade Federal do Maranhão, Brasil

E-mail: gysllaynefsg.enfa@gmail.com

Carolina Freitas do Carmo Rodrigues ORCID: https://orcid.org/0000-0003-1364-8773

Universidade Federal do Tocantins, Brasil E-mail: carolalineppgecs@gmail.com

Maitê da Veiga Feitoza Borges Silva ORCID: https://orcid.org/0000-0001-6117-8775

Universidade Federal do Tocantins, Brasil E-mail: maite.vfbs@gmail.com

Rhavenna Thais Silva Oliveira ORCID: https://orcid.org/0000-0001-8380-6867

Universidade Federal do Tocantins, Brasil E-mail:rav3na@gmail.com

Ruhena Kelber Abrão Ferreira ORCID: https://orcid.org/0000-0002-5280-6263

Universidade Federal do Tocantins, Brasil E-mail: kelberabrao@gmail.com 


\title{
Resumo
}

Objetivo: Identificar o perfil das mulheres atendidas em uma Unidade Básica de Saúde, a periodicidade de realização do PCCU e os fatores que influenciam a não adesão ao exame. Metodologia: Estudo descritivo, exploratório e quantitativo, realizado em uma Unidade Básica de Saúde do centro de Açailândia/MA com 140 mulheres. Foi aplicado um formulário, contendo variáveis relacionadas ao perfil sócio demográfico, perfil clínico/ginecológico e adesão ao exame. Resultados: Evidenciou-se que 64,3\% das participantes estão na faixa etária entre 15 e 37 anos, $55 \%$ são pardas, $57,9 \%$ concluíram o ensino médio, 53,6\% são casadas, $67,1 \%$ se ocupam das atividades do lar e $60 \%$ não possuíam renda mensal. A maioria $(84,3 \%)$ têm até 3 filhos, iniciaram a atividade sexual entre 11 e 18 anos $(69,3 \%)$, possuíam parceiro fixo $(96,4 \%)$ e $45,7 \%$ informam não usar o preservativo nas relações sexuais. Quanto a realização do PCCU $85 \%$ já haviam realizado alguma vez, sendo a periodicidade mais frequente de 1 ano $(52,9 \%)$ e, em média, $14,3 \%$ relataram não realizar o exame preventivo conforme o recomendado por motivo de vergonha ou falta de tempo. Conclusão: Sabe-se que conhecer e avaliar o perfil das mulheres atendidas em uma UBS contribui com o conhecimento do perfil populacional, corroborando com a redução dos índices de câncer do colo do útero. Diante disso, o investimento em educação e capacitação em saúde, tanto dos profissionais quanto das pacientes, é fundamental, além de fornecer assistência à saúde de forma abrangente, conhecendo o perfil da população e, assim, a maior cobertura dos programas de rastreamento, promoção, prevenção e educação em saúde, incluindo também estratégias de enfrentamento da doença reduzindo, dessa forma, a sua incidência e a mortalidade causadas pelo câncer de colo do útero.

Palavras-chave: Câncer do colo do útero; Rastreamento; Atenção primária à saúde; Assistência de enfermagem.

\begin{abstract}
Objective: To identify the profile of women attended at a Basic Health Unit, the frequency of the PCCU and the factors that influence non-adherence to the exam. Methodology: Descriptive, exploratory and quantitative study, carried out in a Basic Health Unit in the center of Açailândia / MA with 140 women. A form was applied, containing variables related to the socio-demographic profile, clinical / gynecological profile and adherence to the exam. Results: It was evidenced that $64.3 \%$ of the participants are in the age group between 15 and 37 years old, 55\% are brown, $57.9 \%$ have finished high school, $53.6 \%$ are married, $67.1 \%$ are in charge of household activities and $60 \%$ had no monthly income. Most (84.3\%) have up to 3 children, started sexual activity between 11 and 18 years old (69.3\%), had a steady partner $(96.4 \%)$ and $45.7 \%$ reported not using condoms during sexual intercourse . Regarding the performance of the PCCU, $85 \%$ had already performed it, being the most frequent period of 1 year $(52.9 \%)$ and, on average, $14.3 \%$ reported not performing the preventive exam as recommended due to embarrassment or lack of time. Conclusion: It is known that knowing and evaluating the profile of women attended at a BHU contributes to the knowledge of the population profile, corroborating the reduction in cervical cancer rates. Given this, investment in health education and training, both for professionals and patients, is fundamental, in addition to providing comprehensive health care, knowing the profile of the population and, thus, the greater coverage of screening, promotion programs., prevention and health education, also including strategies for coping with the disease, thereby reducing its incidence and mortality from cervical cancer.
\end{abstract}

Keywords: Cervical cancer; Tracking; Primary health care; Nursing assistance.

\section{Resumen}

Objetivo: Identificar el perfil de las mujeres atendidas en una Unidad Básica de Salud, la frecuencia de la UCPC y los factores que influyen en la no adherencia al examen. Metodología: Estudio descriptivo, exploratorio y cuantitativo, realizado en una Unidad Básica de Salud en el centro de Açailândia / MA con 140 mujeres. Se aplicó un formulario que contenía variables relacionadas con el perfil sociodemográfico, perfil clínico-ginecológico y adherencia al examen. Resultados: Se evidenció que el 64,3\% de los participantes se encuentra en el grupo de edad entre 15 y 37 años, el 55\% son morenos, el 57,9\% han terminado el bachillerato, el 53,6\% son casados, el 67,1\% están a cargo de actividades del hogar y $60 \%$ no tenía ingresos mensuales. La mayoría (84,3\%) tiene hasta 3 hijos, inició la actividad sexual entre los 11 y 18 años (69,3\%), tenía una pareja estable (96,4\%) y el 45,7\% informó no usar condón durante las relaciones sexuales. Quanto a realização do PCCU 85\% já haviam realizado alguma vez, sendo a periodicidade mais frequente de 1 ano $(52,9 \%)$ e, em média, $14,3 \%$ relataram não realizar o exame preventivo conforme o recomendado por motivo de vergonha ou falta de tiempo. Conclusión: Se sabe que conocer y evaluar el perfil de las mujeres atendidas en una UBS contribuye al conocimiento del perfil de la población, corroborando la reducción de las tasas de cáncer de cuello uterino. Por ello, la inversión en educación y formación en salud, tanto de profesionales como de pacientes, es fundamental, además de brindar una atención integral de salud, conociendo el perfil de la población y, así, la mayor cobertura de tamizaje, programas de promoción, prevención y educación para la salud, incluyendo también estrategias para afrontar la enfermedad, reduciendo así su incidencia y mortalidad por cáncer de cuello uterino.

Palabras clave: Cáncer de cuello uterino; Seguimiento; Primeros auxilios; Asistencia de enfermería. 


\section{Introdução}

O câncer do colo do útero (CCU) resulta numa multiplicação exagerada e desorganizada das células do colo do útero e caracteriza-se como um problema grave de saúde pública. Com o passar dos anos seus números vem aumentando de maneira assustadora e as projeções de novos casos são desanimadoras, somado à possibilidade de agravamento ao poder invadir estruturas e órgãos contíguos ou à distância (INCA, 2020).

Responsável pelo quarto tipo de câncer mais comum entre as mulheres e com aproximadamente 570 mil casos novos por ano em todo o mundo, o CCU em 2020 pode ter chegado a 16.710 casos novos no Brasil, com 890 no estado do Maranhão. Em 2019, foram 6.596 óbitos por esse tipo de câncer no país. (INCA, 2020).

O CCU, também conhecido por câncer cervical, é uma doença que apresenta um quadro evolutivo bastante lento, mas que acomete, sobretudo, mulheres acima dos 25 anos. O principal agente da enfermidade é o Papiloma Vírus Humano (HPV), que pode infectar também os homens e estar associado ao surgimento do câncer de pênis e orofaringe (INCA, 2020; Villa, 2020).

Este tipo de câncer apresenta-se como a terceira localização primária de incidência após do câncer de mama e de cólon e reto, e a quarta causa de mortalidade por câncer entre as mulheres no Brasil, após do câncer de mama, do sistema respiratório e de cólon e reto. Todavia, seu rastreamento, que consiste em um "processo de identificação de pessoas aparentemente saudáveis que podem estar sob maior risco de doença” (INCA, 2016, p. 13), pode ser feito de maneira rápida, simples e efetiva, sendo a principal forma de prevenção do diagnóstico tardio e, consequente, agravamento (Carvalho, 2014; Barbosa, Ricacheneisky, \& Daudt, 2018).

O método de rastreamento do CCU no Brasil é o exame Preventivo do Câncer de Colo do Útero (PCCU), que no Sistema Único de Saúde (SUS) é realizado na Atenção Primária à Saúde (APS). De acordo com o Ministério da Saúde esse exame deve ser realizado em todas as mulheres a partir dos 25 anos e que já tiveram atividade sexual, apesar disso, a população alvo mantém divergências na literatura, sendo necessária uma análise balanceada e cuidadosa das vantagens e desvantagens para cada mulher. O exame consiste na coleta de material do colo uterino para exame em laboratório, caracterizando-se como um exame simples e barato, porém algumas mulheres ainda resistem em realizá-lo por medo ou pela situação de constrangimento, imaginada por grande parte delas (Silva et al, 2015; INCA, 2016; Barbosa, Ricacheneisky, \& Daudt, 2018).

Neste contexto, torna-se indispensável à atuação de uma equipe multidisciplinar em todas as unidades da APS, auxiliando na prevenção e rastreamento de doenças como o CCU. Nessa perspectiva, o enfermeiro pode ser considerado um verdadeiro pilar para que a ação preventiva possa ser oferecida e de fato conhecida entre as mulheres, principalmente por ações educativas frequentes nas unidades, tanto para a equipe quanto para as usuárias para adesão ao exame PCCU (Carvalho, 2014; da Costa et al., 2017).

Diante do exposto, o objetivo do presente artigo consiste em identificar o perfil das mulheres atendidas em uma Unidade Básica de Saúde (UBS) do município de Açailândia/MA, a periodicidade de realização do PCCU e os fatores que influenciam a não adesão ao exame.

\section{Metodologia}

Trata-se de um estudo descritivo de natureza quantitativa com caráter exploratório que foi realizado na UBS Cikel, localizada no centro de Açailândia/MA. A população do estudo foi constituída de mulheres usuárias do SUS e frequentadoras da UBS referida, no período de 12 a 28 de novembro de 2018, selecionadas aleatoriamente através da captação interna e externa à unidade. 
Foram incluídas na pesquisa as usuárias com idade mínima de 12 anos e máxima de 59 anos que já haviam tido relação sexual, residente no bairro e cadastrada e atendida na UBS escolhida. Foram excluídas do estudo mulheres com deficiência mental que tenha impedimento à compreensão do estudo, gestantes, acamadas ou doentes, que ainda não tenham tido relação sexual.

A cada participante foi aplicado um formulário padronizado com 19 questões de múltipla escolha para obtenção de informações sócia demográficas, reprodutiva e comportamental. O questionário foi comporto por: idade, raça ou cor, grau de escolaridade, estado conjugal, ocupação, renda mensal, número de filhos, idade de início da atividade sexual, uso de preservativo nas relações sexuais, frequência de realização do exame PCCU e fatores que influenciam a não adesão ao exame.

Antes de iniciar a coleta de dados, os responsáveis pela pesquisa entraram em contato com a coordenação da UBS informando sobre o estudo e discutindo o interesse a disponibilidade da unidade em fazer parte do projeto. Após aceite e aprovação no Comitê de Ética e Pesquisa (CEP), os entrevistadores informaram a UBS quanto ao período de coleta.

Ao abordarem as mulheres, na área interna e externa à unidade, os entrevistadores informavam quanto ao estudo e seu objetivo, convidando-as a participarem. As mulheres que aceitavam em participar do estudo eram convidadas para uma sala reservada disponibilizada pelas UBS, onde tinham acesso ao Termo de Consentimento Livro e Esclarecido (TCLE) com adequado tempo para que pudessem decidir em participar ou não da pesquisa, de forma que foi preservado o direito de informação. As jovens com menos de 18 anos teriam que ter o TCLE assinados pelos responsáveis e também o Termo de Assentimento Livre e Esclarecido (TALE).

Os dados coletados e analisados surgiram a partir das respostas obtidas na aplicação do formulário, alimentando um banco de dados em planilha de software: Microsoft Office Excel 2010, com todas as variáveis para determinar a quantidade exata das respostas, sendo analisado por meio de frequência. A análise descritiva e estatística foi realizada à luz da literatura pertinente.

\section{Resultados e Discussão}

A população deste estudo foi constituída por 140 mulheres, sendo que 64,3\% possuíam entre 15 e 37 anos, 55\% se autodeclararam pardas, 57,9\% relataram possuir Ensino Médio completo, 53,6\% afirmaram ser casadas, 67,1\% informaram não possuir ocupação e $60 \%$ não possuíam renda, conforme Tabela 1 a seguir: 
Tabela 1. Perfil socioeconômico das mulheres frequentadoras da UBS Cikel. Açailândia-MA, 2018.

\begin{tabular}{|c|c|c|c|}
\hline Características & Especificações & Qtd & $\%$ \\
\hline \multicolumn{4}{|l|}{ Faixa Etária } \\
\hline & $15-37$ & 90 & 64,3 \\
\hline & $38-59$ & 50 & 35,7 \\
\hline \multicolumn{4}{|l|}{ Raça/cor } \\
\hline & Branca & 37 & 26,4 \\
\hline & Parda & 77 & 55 \\
\hline & Negra & 26 & 18,6 \\
\hline \multicolumn{4}{|l|}{ Escolaridade } \\
\hline & Não alfabetiza & 4 & 2,9 \\
\hline & Ensino Fundamental Incompleto & 35 & 25,0 \\
\hline & Ensino Médio Completo & 81 & 57,9 \\
\hline & Nível Superior Completo & 10 & 7,1 \\
\hline & Nível Superior Incompleto & 10 & 7,1 \\
\hline \multicolumn{4}{|l|}{ Estado Civil } \\
\hline & Solteira & 34 & 24,3 \\
\hline & Casada & 75 & 53,6 \\
\hline & União Estável & 23 & 16,4 \\
\hline & Separada & 8 & 5,7 \\
\hline \multicolumn{4}{|l|}{ Ocupação } \\
\hline & Não possuem (do lar, doméstica, estudante, etc...) & 94 & 67,1 \\
\hline & $\begin{array}{l}\text { Cargos de nível fundamental (cabeleireira, faxineira, lavradora, } \\
\text { etc...) }\end{array}$ & 10 & 7,1 \\
\hline & $\begin{array}{l}\text { Cargos de nível médio (caixa, recepcionista, secretária, } \\
\text { vendedora, etc...) }\end{array}$ & 23 & 16,4 \\
\hline & Cargos de nível técnico (técnico de Enfermagem...) & 4 & 2,9 \\
\hline & $\begin{array}{l}\text { Cargos de nível superior (administradora, contadora, assistente } \\
\text { social, etc...) }\end{array}$ & 5 & 3,6 \\
\hline & Aposentadas & 4 & 2,9 \\
\hline \multicolumn{4}{|l|}{ Renda Mensal } \\
\hline & Sem renda & 84 & 60,0 \\
\hline & Menos de 1 salário mínimo & 15 & 10,7 \\
\hline & De 1 a 3 salários mínimos & 41 & 29,3 \\
\hline & Mais de 3 salários & 0 & 0,0 \\
\hline
\end{tabular}

Fonte: Próprios Autores.

As mulheres entrevistadas possuíam entre 15 a 59 anos de idade. Apesar da faixa etária encontrada, isso não representa, necessariamente, que elas realizam o exame PCCU conforme o preconizado. O MS orienta que o início da coleta do exame deve ser aos 25 anos para as mulheres que já tiveram ou têm atividade sexual, porém afirma que o início mais precoce representaria um significativo aumento de diagnóstico de lesões de baixo grau, desde que não realizado de forma indiscriminada (INCA, 2018). O exame deve ser realizado até os 64 anos de idade, sendo o pico de incidência entre os 45 e 50 anos (INCA, 2018), porém segundo o Instituto Oncoguia (2017), há ainda quem comece a se preocupar somente aos 50.

A prevalência da raça parda (55\%) na amostra vai de encontro com a maior concentração dessa raça na população nacional. De acordo com a Pesquisa Nacional por Amostra de Domicílios Contínua (PNAD, 2019), 46,8\% dos brasileiros se autodeclaram como pardos. Em estudo realizado no estado do Maranhão que objetivou a caracterização do perfil epidemiológico do CCU no estado, identificou que em todos os anos avaliados a raça parda foi a mais afetada, porém a raça não caracteriza-se um fator de risco, visto que maior parte da população se autodeclara pertencente à essa raça (de Sá et al., 2020).

No quesito escolaridade, a maioria (57,9\%) afirmou possuir ensino médio completo, havendo destaque para apenas 2,9\% não serem alfabetizadas. No Brasil, a alta prevalência e mortalidade por CCU também estão associadas ao baixo nível socioeconômico, pois enfrentam barreiras de acesso à rede de serviços de saúde e dificuldades na detecção (rastreamento) e 
tratamento, sendo a baixa escolaridade um risco epidemiológico relativo para o desenvolvimento desse tipo de câncer (Lamarca \& Vettore, 2013; da Costa et al., 2017; de Sá et al, 2020).

Para além da prevenção da mortalidade, a escolaridade está diretamente ligada a práticas de autocuidado e melhores condições de saúde, que são influenciadas diretamente pelo entendimento, compreensão da magnitude e acesso a informações de saúde. Estudos de revisão integrativa realizados por Silva et al. (2020) e Silva et al. (2020) evidenciaram que diferentes níveis socioeconômicos e de escolaridade estão diretamente relacionados com o nível baixo de letramento em saúde e acerca dos meios de prevenção do CCU. Assim, educação permanente em saúde e atividades educativas acessíveis junto às mulheres são essenciais para que elas entendam a importância do diagnóstico precoce.

Quanto ao estado civil, 70\% relataram estar em uma relação afetiva, apesar de não ter sido avaliado a orientação sexual das participantes. Medeiros et al. (2015) afirma que independente do estado civil, a existência de prática sexual regular é incentivador para as mulheres frequentarem os serviços de saúde, tornando-se uma oportunidade de contato, orientação e rastreio. Vale ressaltar que $80 \%$ das mulheres sexualmente ativas irão contrair a infecção pelo HPV, maior causador do CCU, reafirmando a necessidade de rastreio dessas mulheres (da Costa et al., 2017).

Em relação a avaliação da ocupação e da renda mensal das entrevistadas, que revelou que $67,1 \%$ não possuíam ocupação remunerada e 60\% não possuíam renda, apresentando uma equivalência em relação ao estudo de Ribeiro et al (2015). Um estudo, realizado na cidade de Vitória, apontou que as mulheres que trabalham em casa ou que não possuem ocupação tenham menos autonomia sobre os cuidados relacionados à saúde, já as trabalham fora de casa apresentam ações de maior autocuidado em relação ao PCCU, já que elas possuem maior acesso à informações estando em contato com outras mulheres, estimulando as práticas de prevenção à saúde (Mascarello et al, 2012). Porém, como já discutido, o baixo nível socioeconômico pode dificultar o acesso aos serviços de saúde, ainda mais quanto à prevenção, e adesão ao rastreamento, caracterizando-se também como um risco epidemiológico relativo para o desenvolvimento desse tipo de câncer (de Sá et al, 2020).

Apesar disso, o SUS garante o acesso gratuito e acessível às medidas de prevenção e diagnóstico a todas as mulheres pela APS desde 1984, quando foi lançado o Programa de Assistência Integral à Saúde da Mulher, que possuía em sua base programática ações de prevenção ao CCU. Em 1998, o MS instituiu o Programa Nacional de Combate ao Câncer de Colo do Útero com estruturação da rede assistencial, estabelecimento de um sistema de informação nacional e mecanismos de captação e mobilização das mulheres. E em caso da necessidade de acompanhamento especializado, assim como de tratamento, o SUS também garante a continuidade do serviço (INCA, 2020).

Mesmo com todo o acesso à saúde disponível pelo SUS, os aspectos socioeconômicos como renda, ocupação e nível de escolaridade, influenciam fortemente na tomada de decisões das mulheres por estarem mais expostas a infecção pelo HPV por apresentar dificuldade para compreender os fatores de vulnerabilidade ao câncer cervical (Tadesse, 2015).

A análise do perfil sexual e ginecológico das mulheres evidenciou que $84,3 \%$ possuía nenhum a três filhos, $69,3 \%$ relataram ter iniciado a atividade sexual entre 11 a 18 anos com parceiro fixo, 49,3\% com namorado e 48,6\% com marido, 96,4\% informaram possuir parceiro fixo, 45,7\% não fazem uso de preservativos, 85,7\% afirmaram já ter apresentado alguma IST, porém, apesar das poucas respostas, 7,9\% não soube informar qual, conforme Tabela 2 abaixo. 
Tabela 2. Perfil sexual e ginecológico das mulheres freqüentadoras da UBS Cikel. Açailândia-MA, 2018.

\begin{tabular}{|c|c|c|c|}
\hline Características & Especificações & Qtd & $\%$ \\
\hline \multicolumn{4}{|c|}{ Número de filhos } \\
\hline & 0 a 3 & 118 & 84,3 \\
\hline & 4 a 6 & 18 & 12,9 \\
\hline & 7 a 9 & 4 & 2,9 \\
\hline \multicolumn{4}{|c|}{ Idade de início da atividade sexual } \\
\hline & 11 a 18 & 97 & 69,3 \\
\hline & 19 a 32 & 43 & 30,7 \\
\hline \multicolumn{4}{|l|}{ Com quem? } \\
\hline & Namorado & 69 & 49,3 \\
\hline & Amigo & 2 & 1,4 \\
\hline & Marido & 68 & 48,6 \\
\hline & Outro & 1 & 0,7 \\
\hline \multicolumn{4}{|l|}{ Parceiro fixo } \\
\hline & Sim & 135 & 96,4 \\
\hline & Não & 5 & 3,6 \\
\hline \multicolumn{4}{|c|}{ Usa preservativos } \\
\hline & Sim & 45 & 32,1 \\
\hline & Não & 64 & 45,7 \\
\hline & Às vezes & 31 & 22,1 \\
\hline \multicolumn{4}{|c|}{ Já apresentou alguma DST } \\
\hline & Não & 120 & 85,7 \\
\hline & Sim & 20 & 14,3 \\
\hline \multicolumn{4}{|l|}{ Se sim, qual? } \\
\hline & Não informar & 11 & 7,9 \\
\hline & Candidíase & 7 & 5,0 \\
\hline & Tricomaníase & 2 & 1,4 \\
\hline
\end{tabular}

Fonte: Próprios Autores.

Sobre o número de filhos, em que maioria (84,3\%) possuía nenhum a três, estudo realizado por Melo et al. (2019) revelou que o conhecimento de mulheres sobre o CCU é significativamente influenciado pela quantidade de filhos que a mulher possui, sendo que quanto menor a quantidade de filhos, melhor o conhecimento. Essa realidade deve-se a sobrecarga com o cuidado dos filhos e demais afazeres, dificultando o autocuidado, como a busca por medidas preventivas, como o exame PCCU (Melo et al., 2019)

O início da atividade sexual precoce foi identificado em nossos achados, ao ponto que 69,3\% começaram entre 11 e 18 anos. A atividade sexual precoce caracteriza-se como um fator de risco clínico e epidemiológico para o CCU, já que a precocidade está vinculada ao não uso de preservativos e, consequente, infecção por IST, como o HPV (Vimar, Braga, \& Vianna, 2020). Além disso, a imaturidade dos tecidos genitais do colo do útero de adolescentes está mais propícia às IST (Brito et al., 2021).

A presença de parceiros fixos foi destaque na pesquisa, tanto para início da atividade sexual com namorado e marido, com 49,3\% e 48,6\% respectivamente, quanto para o presente momento com 96,4\%. A situação conjugal casada/em união estável e a presença de um relacionamento fixo estão associados ao não uso de preservativo, principalmente pela recusa do homem, a falsa percepção de segurança pelo afeto existente e as condições socioeconômicas, expondo as mulheres às IST e, consequentemente, ao HPV e ao CCU (Barbosa et al., 2019).

Essa realidade é confirmada ao ponto que 45,7\% afirmam não fazer uso de preservativo. Esse dado deve-se ao fato da dificuldade existente e tão debatida do uso do preservativo nas relações fixas e, principalmente, heterossexuais. Mesmo a entrega gratuita do preservativo tendo início em 1994, sua associação com orientação adequada é frágil, não eliminando a prática sexual desprotegida e as barreiras culturais, sociais e emocionais envolvidas, sendo necessário ir além do 
aconselhamento, estimulando o empoderamento feminino, já que o preservativo de maior uso é o externo, investindo também em distribuição e aconselhamento sobre o preservativo interno (Rodrigues, 2020).

Apesar do início precoce da vida sexual pela maioria das mulheres e o não uso de preservativo mesmo nas relações fixas, apenas 14,3\% disseram ter apresentado alguma IST, sendo que apenas 6,4\% souberam informar qual (5\% candidíase e 1,4\% tricomaníase). Estudo realizado por Barbosa et al. (2019), que avaliou os fatores relacionados ao não uso do preservativo e a prevalência de IST, associou a maior prevalência de IST à presença de parceiro e/ou relacionamento fixo devido ao não uso do preservativo. O baixo percentual de relatos de diagnóstico pode ser justificado pela ausência de diagnostico prévio e o estigma e preconceito em afirmar possuir ou ter histórico de IST.

Ao analisar a realização do exame PCCU, foi evidenciado que $85 \%$ das mulheres já haviam realizado o exame, com maior frequência a cada ano (52,9\%). A maioria $(43,6 \%)$ das mulheres não soube dizer qual o motivo a impediram de realizar o exame, e daquelas que souberam, houve destaque para a falta de tempo e para o sentimento de vergonha, ambos com 14,3\%. Quanto ao acesso à informação sobre o exame, $75 \%$ afirmaram já ter recebido, sendo $70 \%$ proveniente de profissionais de saúde e 5\% dos meios de comunicação, mas, ainda assim, 95,7\% gostariam de mais informações, conforme Tabela 3 abaixo:

Tabela 3. A realização do PCCU das mulheres frequentadoras da UBS Cikel. Açailândia-MA, 2018.

\begin{tabular}{|c|c|c|c|}
\hline Características & Especificações & Qtd & $\%$ \\
\hline \multicolumn{4}{|c|}{ Você já realizou o exame Papanicolau? } \\
\hline & Não & 21 & 15,0 \\
\hline & Sim & 119 & 85,0 \\
\hline \multicolumn{4}{|c|}{ Com que frequência? } \\
\hline & Nunca realizou & 21 & 15,0 \\
\hline & 6 em 6 meses & 18 & 12,9 \\
\hline & 1 vez por ano & 74 & 52,9 \\
\hline & A cada 2 anos & 8 & 5,7 \\
\hline & Mais de 3 anos & 13 & 9,3 \\
\hline & Outros & 6 & 4,3 \\
\hline \multicolumn{4}{|c|}{ Qual motivo que impediu de realizar o exame? } \\
\hline & Falta de tempo & 20 & 14,3 \\
\hline & Acesso ao serviço de saúde & 14 & 10,0 \\
\hline & Medo do procedimento & 4 & 2,9 \\
\hline & Medo de descobrir o câncer & 2 & 1,4 \\
\hline & Vergonha & 20 & 14,3 \\
\hline & Desinteresse & 19 & 13,6 \\
\hline & N/A & 61 & 43,6 \\
\hline \multicolumn{4}{|c|}{ Já recebeu informações sobre o exame? } \\
\hline & Não & 35 & 25,0 \\
\hline & Sim & 105 & 75,0 \\
\hline & Profissional de saúde & 98 & 70,0 \\
\hline & Televisão/rádio/outros & 7 & 5,0 \\
\hline \multicolumn{4}{|c|}{ Gostaria de ter mais informações sobre o exame? } \\
\hline & Sim & 134 & 95,7 \\
\hline & Não & 6 & 4,3 \\
\hline
\end{tabular}

Fonte: Próprios Autores. 
A maioria (85\%) das mulheres afirmou já ter realizado o exame PCCU. Embora o quantitativo apresentado esteja em concordância com a cobertura de, no mínimo, 80\% da população-alvo (mulheres entre 25 e 64 anos), defendida pela OMS, este achado não pode ser considerado como representativo, visto que não foi avaliado o quantitativo de mulheres atendidas na unidade ou na Rede de Atenção do município, conforme o número de mulheres que participaram do presente estudo, e não foram avaliadas apenas mulheres na faixa etária alvo do exame. Ainda assim, ressalta-se a importância da realização do exame PCCU, visto que pode reduzir em até $90 \%$ a incidência do CCU (INCA, 2018).

Apesar disso, 15\% das mulheres entrevistadas nunca haviam realizado o exame, mesmo frequentando a unidade da APS. Em 2018, a Sociedade Brasileira de Oncologia Clínica divulgou que 52\% das mulheres brasileiras não fazem o exame PCCU (SBOC, 2018). Mesmo não ter sido associado essa categoria à faixa etária alvo do exame, ressaltamos a importância e necessidade da abordagem pelos profissionais de saúde, com investigação dos fatores de risco e oferta de acesso ao rastreamento, garantindo organização, integralidade e qualidade no rastreamento, assim como busca ativa das mulheres (da Costa et al., 2017).

A frequência de realização do exame chamou a atenção, visto que 52,9\% afirmaram fazer uma vez por ano, 12,9\% de 6 em 6 meses, 9,3\% depois de mais de 3 anos, 5,7\% a cada dois anos e 4,3\% em outro intervalo. Devido à longa evolução da doença, o MS preconiza que o exame pode ser realizado a cada três anos, após dois ou três resultados anuais sem alterações. Para maior segurança do diagnóstico, os dois primeiros exames devem ser anuais (INCA, 2016). Segundo Conde, Lemos e Ferreira (2018), a não realização do PCCU de rotina foi identificado como um importante fator de risco, uma vez que isso contribui para a vulnerabilidade a neoplasia.

A frequência anual do exame PCCU não apresenta evidências de maior efetividade do que com intervalo a cada três anos, caso não haja alteração, e sua realização demonstra a necessidade de maior controle por parte do serviço de saúde, como por meio do agendamento de retorno e/ou carta-convite e busca ativa das faltosas e das ausentes pelos Agentes Comunitários de Saúde. Demandas desorganizadas e oportunísticas, como a observada, não reduz a morbimortalidade de forma eficaz, provocando mulheres rastreadas de forma excessivas em detrimento de nenhum ou rastreio inadequado, principalmente das mulheres de maior risco (Lima, 2018).

A maioria das mulheres $(43,6 \%)$ não soube apontar uma dificuldade específica para não realizarem o exame PCCU, o que pode ser consequência do desconhecimento da importância e necessidade da realização frequente do exame de rastreamento para o CCU. Das mulheres que expressaram um empecilho, 14,3\% justificaram como a falta de tempo e $14,3 \%$ a vergonha, tendo também 13,6\% por desinteresse, $10 \%$ pela dificuldade de acesso ao serviço de saúde, 2,9\% por medo do procedimento e $1,4 \%$ por medo de descobrir o câncer.

O exame PCCU é indolor, de baixo custo, de alta eficácia e é realizado em consultório, garantindo a privacidade da paciente, de forma rápida e simples, não sendo necessário usar anestesia ou qualquer outro tipo de medicamento ou preparo anterior. A sobrecarga da mulher já foi discutida e soma-se ao exposto a pressão estética e o tabu quanto à genitália, ao justificarem não realizar um exame de autocuidado por vergonha, exigindo dos profissionais de saúde comportamento ético e técnico no intuito de proteger a intimidade da mulher e assegurar o acesso à saúde (da Costa et al., 2017; Garcia et al., 2021).

$\mathrm{O}$ acesso à informação foi verificado quando $75 \%$ das mulheres afirmaram já terem recebido informações sobre a importância da realização do exame PCCU, sendo $70 \%$ proveniente dos profissionais de saúde e 5\% dos meios de comunicação (televisão, rádio e outros). Ainda que maioria afirmasse acesso à informação, $15 \%$ das mulheres que estavam frequentando a unidade de saúde negaram.

A informação é ferramenta essencial para maior alcance do rastreio e quebra de paradigmas, devendo ser realizada de forma frequente e com linguagem acessível a todos, independentemente da escolaridade e classe social, visto que a falta de conhecimento adequado interfere diretamente na realização e adesão ao exame. Destaca-se nesse contexto a necessidade de 
informações para além da realização do exame, mas também para a finalidade, autoconhecimento e para outros hábitos de autocuidado, como o uso do preservativo e o conhecimento sobre IST (Garcia et al., 2021).

A enfermagem desempenha papel importante na prevenção e deteç̧ão do CCU ao ter na Resolução COFEN $n^{\circ}$ 385/2011 a regulamentação para a realização do exame PCCU, além de sensibilizar, promover a integração com a equipe e incentivar as mulheres a fazerem o exame de rastreio por meio de programas educativo, sendo responsáveis também pela coleta do material e estímulo do autoconhecimento do corpo e da sexualidade, familiarizando a mulher ao ambiente e ao exame e minimizando sentimentos negativos (Sá, et al, 2015). Além disso, a enfermagem também é responsável por treinar a equipe, orientar o indivíduo, a família e a comunidade sobre os fatores de risco do câncer e sobre as formas de prevenção, proporcionando meios concretos e seguros para o tratamento e reabilitação do indivíduo, se necessário (Sá et al, 2015; da Costa et al., 2017).

Ao serem questionadas se gostariam de receber mais informações sobre o exame, 95,7\% das mulheres disseram que "sim". Segundo o INCA (2020), o êxito das ações de rastreamento depende dos seguintes pilares: informar e mobilizar a população e a sociedade civil organizada; alcançar a meta de cobertura da população alvo; garantir acesso a diagnóstico e tratamento; garantir a qualidade das ações e monitorar e gerenciar continuamente as ações. Soma-se ao exposto o papel da mídia, que se caracteriza como ferramenta importante ao acesso à informação pela população, com consequente quebra de tabus e preconceitos quanto ao rastreamento e às IST, proporcionando acesso às formas de prevenção, os fatores de riscos e empoderamento das mulheres para o autocuidado (Silva, 2019; da Silva \& Schumacher, 2020).

$\mathrm{O}$ exame de rastreamento do $\mathrm{CCU}$ possui a vantagem de poder ser realizado durante as consultas de planejamento familiar, de pré-natal, ginecológica entre outras que tratam da saúde da mulher. Diante disso, o exame é realizado oportunamente nas mesmas mulheres que frequentam os serviços de saúde. Realizar o controle do CCU ainda representa um dos maiores desafios para a saúde pública, principalmente pelos estigmas envolvidos (da Costa et al., 2017; da Silva \& Schumacher, 2020).

\section{Conclusão}

Sabe-se que conhecer e avaliar o perfil das mulheres atendidas em uma UBS contribui com o conhecimento do perfil populacional, corroborando com a redução dos índices de CCU da região, pois haverá o direcionamento dos programas voltados para a atenção à saúde dessa população, além de informações sobre a doença e sua epidemiologia, favorecendo também o rastreamento e diagnóstico precoce do CCU.

Por meio desse estudo, foi possível observar que a maioria das mulheres possuía entre 15 e 37 anos, ensino médio completo, nenhum a três filhos e parceiro fixo; eram pardas e casadas; não possuíam ocupação e renda; haviam iniciado a atividade sexual entre 11 e 18 anos, não usavam preservativo e possuíam histórico de IST. Referente ao PCCU, maioria relatou já ter realizado o exame, com frequência de uma vez ao ano, ter recebido informação sobre por profissionais de saúde e gostariam de mais informação, sendo que a falta de tempo e a vergonha foram os maiores obstáculos para realização.

Soma-se ao exposto que por ser causado por uma IST comumente disseminada, o HPV, e por ser um câncer assintomático, na maior parte do tempo, com rastreio considerado invasivo, o diagnóstico do CCU ocorre muitas vezes de forma tardia, ressaltando a importância da informação de qualidade, como os principais fatores de risco e dúvidas em relação à doença, conscientizando-as quanto à importância do exame, e ainda na realização do mesmo, encaminhando-as sempre que necessário a um especialista para um tratamento específico e recuperação da saúde. Destaca-se, assim, a importância do profissional de Enfermagem, tanto na educação da população para acesso ao exame e aos cuidados, quanto para a realização do exame. 
Diante disso, o investimento em educação e capacitação em saúde, tanto dos profissionais quanto das pacientes, é fundamental, além de fornecer assistência à saúde de forma abrangente, conhecendo o perfil da população e, assim, a maior cobertura dos programas de rastreamento, promoção, prevenção e educação em saúde, incluindo também estratégias de enfrentamento da doença reduzindo, dessa forma, a sua incidência e a mortalidade causadas pelo CCU.

Como fatores limitantes encontrados neste estudo destacamos: a não realização do trabalho exclusivo com um público alvo do rastreamento, não ter sido calculada uma amostra representativa da população feminina da unidade, não ter pesquisado a orientação sexual das entrevistas e não ter questionado quanto à existência de alterações prévias. Diante disso, sugerimos que mais estudos sejam realizados, garantindo e incentivando a elaboração de políticas públicas e propostas de intervenção orientando as mulheres sobre a importância da realização do exame, a fim de aumentar a informação e a adesão ao mesmo, evitando e/ou minimizando, assim, possíveis agravos provenientes da não realização do exame e a redução da incidência de diagnóstico tardio do CCU.

\section{Referências}

Barbosa, K. F., Batista, A. P., Nacife, M. B. P. S. L., Vianna, V. N., Oliveira, W. W. D., Machado, E. L., \& Machado-Coelho, G. L. L. (2019). Fatores associados ao não uso de preservativo e prevalência de HIV, hepatites virais B e C e sífilis: estudo transversal em comunidades rurais de Ouro Preto, Minas Gerais, entre 2014 e 2016. Epidemiologia e Serviços de Saúde, 28, e2018408.

Barbosa, A. P., Ricacheneisky, L. F., \& Daudt, C. (2018). Prevenção e rastreamento de neoplasias femininas: mama e colo do útero. Acta Méd, 39(2), 335-45.

Brito, A. B., Fernando, J., Pereira, S., Batalha, M. N., Ferreira, W. M., \& da Silva Lopes, D. I. (2021). Avaliação do conhecimento de estudantes tocantinenses do Ensino Médio de uma escola pública no norte do estado sobre o exame de rastreio do HPV e a principal via de transmissão. Facit Business and Technology Journal, 1(22).

Carvalho, A. P. (2014) Avaliação da qualidade da cobertura do rastreamento do câncer do colo do útero realizado no município de Angicos-RN. Monografia apresentada ao Instituto Nacional do Ensino Superior e Pesquisa e ao Centro de Capacitação Educacional. Recife-PE.

COFEN. Conselho Federal de Enfermagem. Resolução n 385 de 18 de julho de 2011 que normatiza a execução, pelo Enfermeiro, da coleta de material para colpocitologia oncótica pelo método de Papanicolau. http://www.cofen.gov.br/resoluo-cofen-n-3852011_7934.html.

Conde, C. R., Lemos, T. M. R. \& Ferreira, M. L. S. M. (2018). Características sociodemográficas, individuais e programáticas de mulheres com câncer de colo do útero. Enfermería Global, 49, 359-369.

da Costa, F. K. M., Weigert, S. P., Burci, L., \& do Nascimento, K. F. (2017). Os desafios do enfermeiro perante a prevenção do câncer do colo do útero.

de Sá, R. L., Rodrigues, Y. A., de Oliveira, E. H., \& Britto, M. H. M. (2020). Mortalidade por neoplasia maligna do colo do útero no estado do Maranhão: perfil epidemiológico e tendência. Research, Society and Development, 9(4), e13942876-e13942876.

Da Silva, E. G., dos Santos, R. K., de Alencar Oliveira, L., Leal, B. M. N., de Lima Carvalho, M., Silva, B. G. S., \& dos Santos Moura, N. (2020). Letramento em saúde e prevenção do câncer do colo de útero. Brazilian Journal of Development, 6(7), 43439-43448.

da Silva, I. M. L., \& Schumacher, B. (2020). Conhecimento e sentimentos das mulheres com diagnóstico de câncer de colo de útero. Redes-Revista Interdisciplinar do IELUSC, 1(3), 85-94.

dos Santos Silva, M. A., Teixeira, É. M. B., Ferrari, R. A. P., Cestari, M. E. W., \& Cardelli, A. A. M. (2015). Fatores relacionados a não adesão à realização do exame de Papanicolau. Revista da Rede de Enfermagem do Nordeste, 16(4), 532-539.

Garcia, M., Campos, G. K. P., Patrício, J. A. L., Rodrigues, L. A., \& Rodrigues, A. D. F. M. (2021). Identificação dos fatores que interferem na baixa cobertura do rastreio do câncer de colo uterino através das representações sociais de usuárias dos serviços públicos. Brazilian Journal of Health Review, 4(1), $1462-1477$.

Instituto ONCOGUIA (2018). Câncer de mama atinge cada vez mais mulheres abaixo de 30 anos, dizem especialistas. http://www.inca.gov.br/wps/wcm/connect/tiposdecancer/site/home/colo_utero.

INCA (2020). Instituto Nacional de Câncer José Alencar Gomes da Silva. Colo do útero. http://www.inca.gov.br/wps/wcm/connect/tiposdecancer/site/home/colo_utero.

INCA (2018). Instituto Nacional de Câncer José Alencar Gomes da Silva. Diretrizes Brasileiras para o Rastreamento do Câncer do Colo do Útero. Sumário Executivo para a Atenção Básica.

INCA (2016). Instituto Nacional de Câncer José Alencar Gomes da Silva. Coordenação de Prevenção e Vigilância. Divisão de Detecção Precoce e Apoio à Organização de Rede. Diretrizes brasileiras para o rastreamento do câncer do colo do útero. (2a ed.), INCA. 114p.

Lamarca, G., Vettore, M. (2013) Por que algumas mulheres não fazem o exame preventivo de colo de útero? <http://dssbr.org/site/2013/12/por-que-algumasmulheres-nao-fazem-o-exame-preventivo-de-colo-de-utero>. 
Research, Society and Development, v. 10, n. 4, e51710414079, 2021

(CC BY 4.0) | ISSN 2525-3409 | DOI: http://dx.doi.org/10.33448/rsd-v10i4.14079

Lima, S. N. D. S. (2018). Tecnologia para promoção da realização do exame papanicolaou para prevenção do câncer de colo uterino na Atenção Primária. Dissertação (Saúde da Mulher). Universidade Federal do Piauí.

Mascarello, K. C., Silva, N. F., Piske, M. T., Viana, K. C. G., Zandonade, E. \& Amorim, M. H. C. (2012). Perfil Sociodemográfico e Clínico de Mulheres com Câncer do Colo do Útero Associado ao Estadiamento Inicial. Revista Brasileira de Cancerologia, 58(3), 417-426.

Medeiros, T. (2015) Conhecimento e percepção de mulheres quanto ao exame preventivo para o câncer de colo do útero. REBES, 5 (4), 09-16.

Melo, E. M. F. D., Linhares, F. M. P., Silva, T. M. D., Pontes, C. M., Santos, A. H. D. S., \& Oliveira, S. C. D. (2019). Câncer cervico-uterino: conhecimento, atitude e prática sobre o exame de prevenção. Revista Brasileira de Enfermagem, 72, 25-31.

PNAD (2019). Pesquisa Nacional por Amostra de Domicílios. Instituto Brasileiro de Geografia e Estatística. Cor ou raça. https://educa.ibge.gov.br/jovens/conheca-o-brasil/populacao/18319-cor-ou-raca.html\#: :text=De\%20acordo\%20com\%20d ados\%20da,1\%25\%20c omo\%20 am arelos $\% 20$ ou\%20ind\%C3\%ADgenas.

Ribeiro, J. F., Silva, A. R. V., Campelo, V., Santos, S. L. D. \& Coêlho, D. M. M. (2015). Perfil sociodemográfico e clínico de mulheres com câncer do colo do útero em uma cidade do Nordeste. Revista Eletrônica Gestão \& Saúde, 6(2), 1367-81.

Rodrigues, C. F. C., Alves, M. M. M., Brustulin, R., \& Ferreira, R. K. A. (2020). Avaliação do Controle do HIV/Aids na Atenção Primária em Palmas/TO. Research, Society and Development, 9(9), e372997126-e372997126.

Sá, M. I., Silva, M. T., Almeida, D., Vieira, B., Lima, T., Conde, C., ... \& Oliveira, T. (2015). Infeções sexualmente transmissíveis e factores de risco nas adolescentes e jovens: Dados de um Centro de Atendimento a Jovens. NASCER E CRESCER-BIRTH AND GROWTH MEDICAL JOURNAL, $24(2), 64-69$.

SBOC. (2018) Sociedade Brasileira de Oncologia Clínica. Notícias. SBOC divulga que 52\% não fazem exame ginecológico preventivo de câncer. https://sboc.org.br/noticias/item/1151-sboc-divulga-que-52-nao-fazem-exame-ginecologico-preventivo-de-cancer.

Silva, M. L., Nunes, J. S. S., de Oliveira, K. S., \& Leite, T. A. S. (2020). Conhecimento de mulheres sobre câncer de colo do útero: Uma revisão integrativa. Brazilian Journal of Health Review, 3(4), 7263-7275.

Silva, R. L. D. (2019) A importância da medicina preventiva na prevenção e diagnostico do câncer de colo útero nas mulheres em idade fértil na Estratégia Saúde da Família-Tenoné II no município de Belém-PA. Dissertação (Saúde da Família). Universidade Federal do Pará, Universidade Aberta do SUS.

Tadesse, S. K. Socio-economic and cultural vulnerabilities to cervical cancer and challenges faced by patients attending care at Tikur Anbessa Hospital: a cross sectional and qualitative study. (2015). BMC Women's Health, 15(75), 1-12.

Villa, L. L. (2020) Vacina papilomavírus humano (HPV): Atualização e perspectivas. Ver Imunizações, 13 (4), $12-15$.

Vimar, A. C. D. A. V., da Silva Braga, A. C., \& Vianna, Y. I. (2020, February). CÂNCER DE COLO UTERINO E HPV. In Simpósio DE Pesquisa E de Práticas Pedagógicas da UGB (No. 8). 